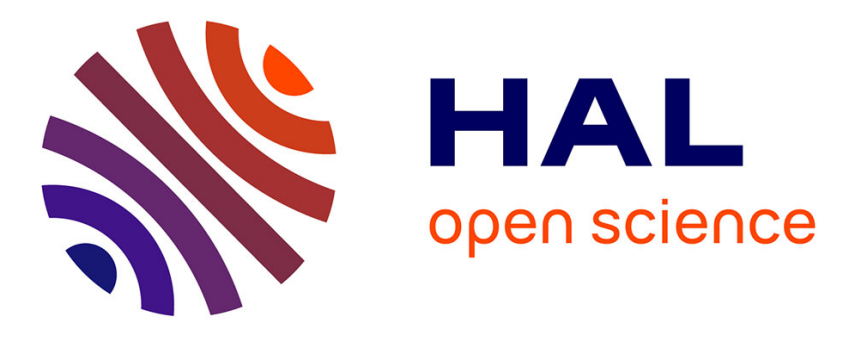

\title{
Light scattering by correlated disordered assemblies of nanoantennas
}

\author{
Eslam El Shamy, Julien Jaeck, Riad Haïdar, Patrick Bouchon
}

\section{To cite this version:}

Eslam El Shamy, Julien Jaeck, Riad Haïdar, Patrick Bouchon. Light scattering by correlated disordered assemblies of nanoantennas. Applied Physics Letters, 2019, 115 (4), pp.041103. 10.1063/1.5097461 . hal-02296871

\section{HAL Id: hal-02296871 \\ https://hal.science/hal-02296871}

Submitted on 25 Sep 2019

HAL is a multi-disciplinary open access archive for the deposit and dissemination of scientific research documents, whether they are published or not. The documents may come from teaching and research institutions in France or abroad, or from public or private research centers.
L'archive ouverte pluridisciplinaire HAL, est destinée au dépôt et à la diffusion de documents scientifiques de niveau recherche, publiés ou non, émanant des établissements d'enseignement et de recherche français ou étrangers, des laboratoires publics ou privés. 


\section{Light scattering by correlated disordered assemblies of nanoantennas}

Eslam El Shamy, ${ }^{1}$ Julien Jaeck, ${ }^{1}$ Riad Haïdar, ${ }^{1,2}$ and Patrick Bouchon ${ }^{1, a)}$

${ }^{1)}$ DOTA, ONERA, Université Paris-Saclay, F-91123 Palaiseau - France

2) École Polytechnique, Département de Physique, 91128 Palaiseau, France

(Dated: 3 July 2019)

Optical nanoantennas are widely used to build absorbing meta-surfaces with applications in photo-detection, solar cells and sensing. Most of the time, the nanoantennas are assembled as a periodic distribution, but there have been various works where disordered arrays are used, either to get rid of diffraction orders or due to a fabrication process that prevents any determined distribution. Here, we investigate both theoretically and experimentally, the unavoidable scattering introduced by such disorders. By introducing a perturbation on the positions of $1 \mathrm{D}$ arrays of metal-insulator-metal (MIM) nanoantennas, the light is scattered rather than increasingly absorbed. The scattering is occurring only in the plane of incidence and on a given spectral range. We show how this scattering can be manipulated from 0 to $55 \%$ of the incoming light.

Optical nanoantennas is a very common building block of metasurfaces. Most of the time, the nanoantennas are assembled as a periodic distribution. However, there have been various works where disordered arrays are used with a large variety of motivations ${ }^{1}$. Among the most obvious reasons, suppressing the periodicity allows getting rid of diffraction orders ${ }^{2,3}$. It can also allow various bottom-up fabrication techniques that are scalable, but prevents any determined distribution, which is very appealing for applications where large areas are needed $^{4-7}$. Analytical models have been developed to describe disordered nanoparticles scattered on a transparent substrate ${ }^{8,9}$. Disorder has also been used to improve performances of optical components ${ }^{10-12}$ or of light extraction $^{13}$, as well as to enhance physical effects such as plasmons localization ${ }^{14,15}$ or surface enhanced Raman spectroscopy ${ }^{16,17}$. The influence of disorder to reduce the scattered fields have been previously reported in the microwaves region, using randomly distributed unit cells $^{18,19}$. Quantifying the diffuse reflection energy is rather challenging, both numerically because it results in huge electromagnetic problems for a given computation accuracy, and experimentally because the diffuse reflection throws in $2 \pi$ steradian and cannot be detected without a high signal to noise ratio experiment. This challenge can be simplified by studying nanoantennas infinite along one direction. Indeed, it results in less-demanding computations while keeping a good numerical accuracy. Besides, a 1D grating scatters light in the plane of incidence increasing the signal, thus reducing the experimental challenge.

Here, we investigate both theoretically and experimentally, the scattering introduced by a correlated disorder in arrays of 1D metal-insulator-metal (MIM) nanoantennas, which are used to build absorbing metasurfaces ${ }^{20-24}$. MIM nanoantennas have previously been used as local absorber or emitter ${ }^{25-27}$, but here, as the disorder is increased, we show that the diffractive orders are progressively disappearing, and the energy is redirected exclu-

\footnotetext{
a)Electronic mail: patrick.bouchon@onera.fr
}
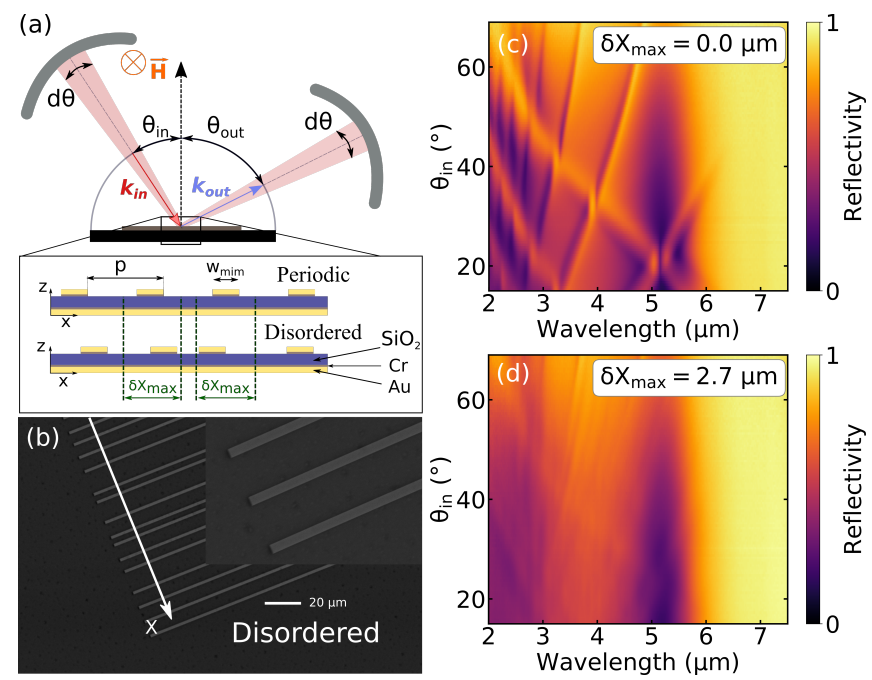

FIG. 1. (a) Experimental configuration used for the angular characterization of the sample. The inset shows the scheme of the two kinds of samples studied: periodic and disordered. In both cases, the geometrical parameters are $\mathrm{w}_{\mathrm{mim}}=1.35 \mu \mathrm{m}$, $\mathrm{h}_{\mathrm{Au}}=0.1 \mu \mathrm{m}, \mathrm{h}_{\mathrm{SiO} 2}=0.3 \mu \mathrm{m}$ and $\mathrm{h}_{\mathrm{Cr}}=5 \mathrm{~nm}$. (b) SEM images of a periodic sample (period $p=7.5 \mu \mathrm{m}$ ) and a disordered sample (disorder parameter $\delta \mathrm{X}_{\max }=0.6 \mu \mathrm{m}$ ). Measured specular reflectivity diagram as a function of the incident angle for (c) a periodic array (period $p=7.5 \mu \mathrm{m})$ and (d) a disordered array (disorder parameter $\delta \mathrm{X}_{\max }=2.7 \mu \mathrm{m}$ ).

sively into scattering. The introduction of diffractive orders in the response of MIM nanoantennas results in a strong coupling between the localized resonance and the diffracted order ${ }^{28,29}$ and it could have been expected that the suppression of the diffraction behavior would bring back the MIM to their original behavior of local absorber with a given absorption effective cross-section. We also show how such metasurfaces can be used to manipulate the amount of scattering on the spectral band of resonance of the antenna.

The geometry of the MIM resonator array studied in the following is shown in Fig. 1(a), it consists of an array of gold ribbons (width $w_{\text {mim }}=1.35 \mu \mathrm{m}$, period $p=7.5 \mu \mathrm{m})$ of $100 \mathrm{~nm}$ thickness defined by an e-beam 

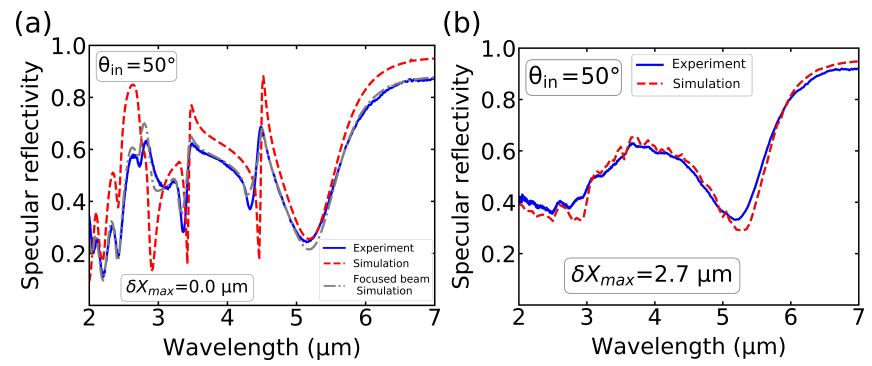

FIG. 2. Theoretical (red dashed line) and experimental (blue continuous line) reflectivity spectra for a TM wave incident with an angle $\theta_{\text {in }}=50^{\circ}$ for (a) the periodic grating and (b) a disordered array (disorder parameter $\delta \mathrm{X}_{\max }=2.7 \mu \mathrm{m}$ ).

lithography and deposited on a continuous layer of silicon dioxide (thickness $300 \mathrm{~nm}$ ) and an optically opaque gold layer (thickness $200 \mathrm{~nm}$ ). A 5-nm adhesion chromium layer is used at both interfaces between silicon dioxide and gold. Disorder is introduced by moving each metallic ribbon from a displacement that is sampled from a uniform probability law with a deviation $\delta \mathrm{X}_{\max }$, which is refered hereafter as the disorder parameter. This disorder parameter gives the maximum possible displacement, and is chosen in the manuscript so as to prevent any overlap between two ribbons. In order to reduce the computation burden, disorder was introduced on a subset of 50 ribbons, which was repeated periodically. A top-view scanning electron microscope image of the periodic and disordered array of MIM ribbons is shown in Fig. 1(b). The samples are experimentally studied with a Fourier transform infrared spectroscope (Bruker Vertex 70v) with a configuration depicted in Fig. 1(a). The incoming light is TM-polarized and focused by a mirror on the sample with an incoming angle $\theta_{\mathrm{in}}$, and the beam collected by a second mirror at an angle $\theta_{\text {out }}$ is detected by a DTGS detector. Both focused beams have a numerical aperture $\mathrm{d} \theta$, which value slightly depends on the angle of incidence, and is typically $10^{\circ}$.

The measured specular reflectivity diagram of the periodic MIM array as a function of the incidence angle is shown in Fig. 1(c). This structure exhibits a fundamental resonance at $\lambda_{r}=5.15 \mu \mathrm{m}$, with several characteristic properties: a high absorption of light, a low quality factor of the resonance $Q \simeq 10$, an independence of the resonance wavelength to the angle of incidence. Since the period of the array is not subwavelength, the reflectivity diagram also shows the various Rayleigh-Wood anomalies (RWA), due to the appearance/disappearance of diffraction orders. The RWA can be suppressed with the introduction of a disorder $\left(\delta \mathrm{X}_{\max }=2.7 \mu \mathrm{m}\right)$, restoring the intrinsic response of the MIM resonator, thus freed of the coupling with diffraction orders (see Fig. 1(d)). This is very appealing since the presence of RWA is known to be detrimental to the absorption efficiency. However, the energy can be either redirected towards absorption or towards the scattering that stems from the disorder arangement.
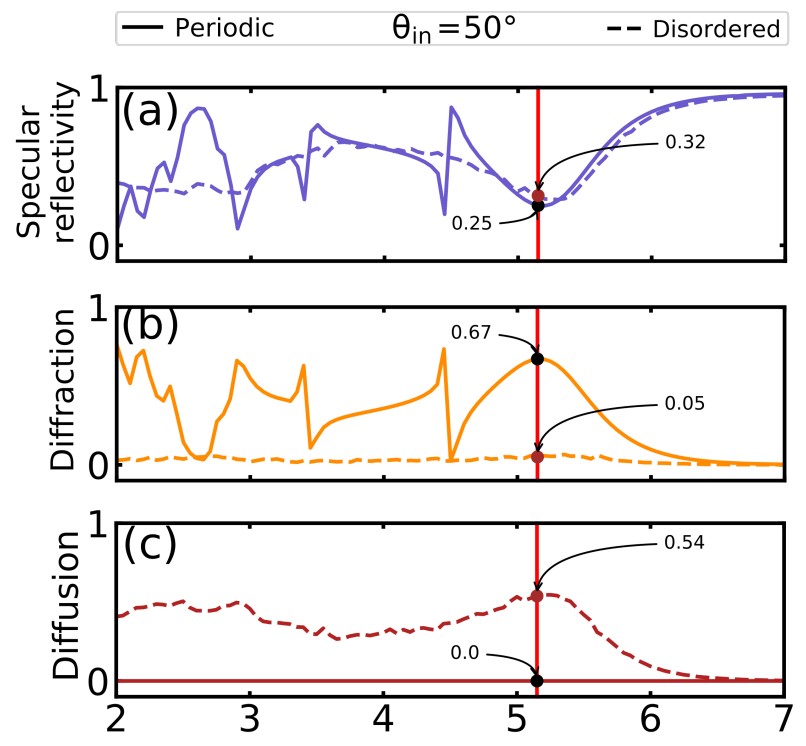

(d) Wavelength $(\mu \mathrm{m})$

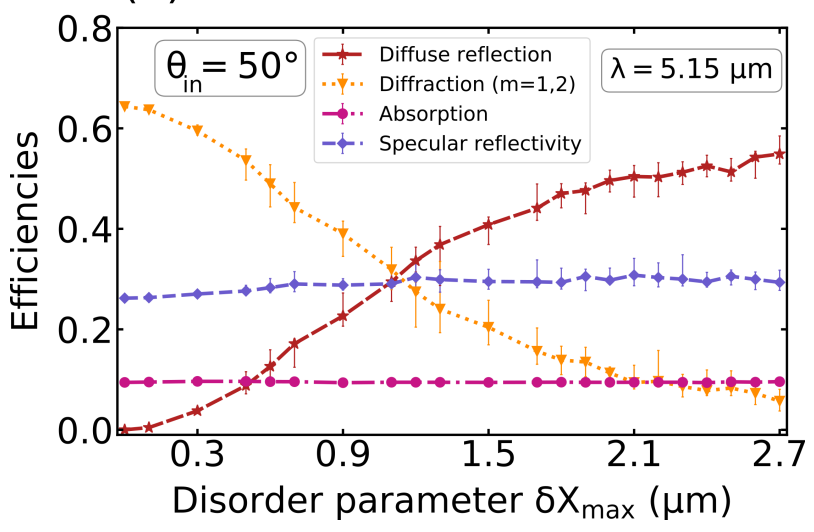

FIG. 3. Computed reflectivity spectra for the periodic array (continuous line) and the disordered array with a disorder parameter $\delta \mathrm{X}_{\max }=2.7 \mu \mathrm{m}$ (dashed line) divided into three parts: (a) the specular reflectivity, (b) the diffracted reflectivity and (c) the scattering, for an angle $\theta_{\text {in }}=50^{\circ}$. (d) Specular reflectivity, diffuse reflection, diffraction and absorption efficiency as a function of the disorder parameter $\delta \mathrm{X}_{\max }$ at $\lambda=5.15 \mu \mathrm{m}$ and $\theta_{\text {in }}=50^{\circ}$. Errorbars represent the data range for the 10 draws and markers indicate the mean values for each data range.

Both samples responses were simulated with an exact Maxwell solver ${ }^{30,31}$. The optical properties of gold are modeled by a Drude model that fits Palik data ${ }^{32}$, and the silicon dioxide is modeled by a Brendel-Bormann model $^{33}$. The theoretical (red line) and experimental (blue line) specular reflectivity spectra are compared in Fig. 2 for an incident angle of $\theta_{\text {in }}=50^{\circ}$. There is a good agreement on the spectral position of the RWA and on the fundamental resonance, but overall, the amplitudes of the RWA are pretty different. In order to get a better agreement, the optical beam numerical aperture has to be taken into account in the simulation with a $d \theta=10^{\circ}$. In that case, a very good agreement between experiment 

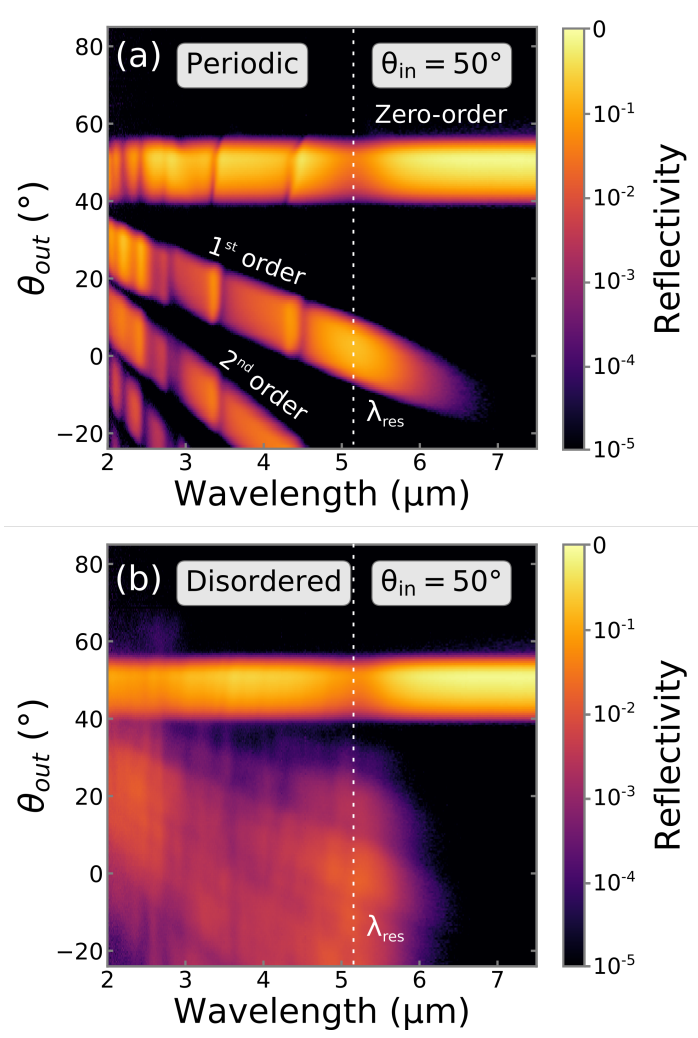

FIG. 4. Measured reflectivity diagram as a function of the scattering angle $\theta_{\text {out }}$ (incidence angle $\theta_{\text {in }}=50^{\circ}$ ) for (a) the periodic grating and (b) the disordered array $\left(\delta \mathrm{X}_{\max }=2.7 \mu \mathrm{m}\right)$.

and simulation (gray curve) is obtained. The various RWA peaks observed in Fig.2(a) are vanishing when considering the disordered array (Fig.2(b)), and there are two minima of reflectivities corresponding to the fundamental resonance of the MIM and its second harmonic at $\lambda_{\text {harmonic }} \simeq 2.6 \mu \mathrm{m}$. At the resonance wavelength, nearly $70 \%$ of the light is diffracted by the periodic array. With the introduction of disorder, the diffracted energy can be redirected into either radiative (diffuse reflection) or nonradiative (absorption) losses since the specular reflectivity $R_{\text {spec }}$ is almost unchanged. There is still a reminiscent diffraction behavior in disordered systems, and in the following, the value of scattering $R_{\text {scatt }}$ does not include the energy scattered in direction similar to diffraction angles $R_{\text {diffracted }}$.

The scattering spectra of the specular reflectivity, the diffracted reflectivity and the diffuse reflection are plotted in Figure 3(a-c) for both the periodic array and the disordered array. The diffraction spectra confirm the vanishing of the RWA, even if around $10 \%$ of the energy is still reflected in the same direction as the diffracted orders. Eventually, a large part of the energy which is no longer diffracted is in fact scattered with a resonance behavior at $\lambda_{r}=5.15 \mu \mathrm{m}$. Below $\lambda_{r}$, there is still an important diffuse reflection $>30 \%$, which could be attributed to the various resonances. On the contrary, there is no longer diffuse reflection for wavelengths above $6.5 \mu \mathrm{m}$, when there is no resonances anymore.

Figure 3(d) shows the influence of the disorder parameter $\delta \mathrm{X}_{\max }$ on the balance of energy between specular reflection, diffraction, diffuse reflection and absorption, which is deduced from the conservation of energy: $A=1-R_{\text {spec }}-R_{\text {diffracted }}-R_{\text {scatt }}$, at the fundamental resonance wavelength of the MIM $\lambda_{r}=5.15 \mu \mathrm{m}$ and for an angle of incidence $\theta_{\text {in }}=50^{\circ}$. The increase of disorder has nearly no influence on the value of absorption and of the specular reflectivity, whereas the diffracted reflectivity decreases monotonously as $\delta \mathrm{X}_{\max }$ grows, and the energy is redistributed almost exclusively in diffuse reflection. In the highest case of disorder, the proportion of diffused reflectivity can reach more than $50 \%$ of total incoming energy. Disorder changes thereby the initially highly diffractive structure $(60 \%)$ into a strongly scattering one. It must be emphasized that even if the absorbed power does not depend on the disorder, each antenna of a given disordered subset absorbs a different amount of the incoming light due to interactions with its neighbors. A given disorder parameter can produce pretty different structures, and for each value of the disorder parameter 10 draws were computed. Errorbars on the Fig. 3(d) represent the data range and the mean value for each data range is given by the marker. The dispersion is higher for intermediate values of the disorder parameter, and could be used to optimize a metasurface with a given scattering behavior.

Measured asymmetric angle resolved reflectivity diagram is presented on Fig. 4(a) for the periodic case for an incidence angle of $\theta_{\text {in }}=50^{\circ}$. The various bands shown on this diagram are the diffraction orders, each of which is following the classic law of grating diffraction $\theta_{\text {out }}=\arcsin \left(\sin \theta_{\text {inc }}-\frac{m \lambda}{d}\right)$. The width of each band is directly linked to the beam numerical aperture $d \theta$ generated by the mirror. As expected, the localized resonance occurring at $\lambda=5.15 \mu \mathrm{m}$ does not depend on the angle of incidence. The dark stripes on each diffraction band are due to the redistribution of energy associated to the appearance or disappearance of a diffracted order (RWA). A similar measured diagram for the highest disordered $\left(\delta \mathrm{X}_{\max }=2.7 \mu \mathrm{m}\right)$ sample is plotted in Fig. $4(\mathrm{~b})$. The specular reflectivity band is nearly unchanged, apart from the vanishing of the RWA features. But for values of $\theta_{\text {out }}$ below $40^{\circ}$, the diffracted orders bands have been replaced by diffuse reflection occurring for every angle. Here, we take advantage of the high numerical aperture $d \theta$ and of the fact that scattering can only occur in the $x z$-plane to obtain a good signal to noise ratio in the measurement. Noteworthily, no scattering is measured above $\theta_{\text {out }}>55^{\circ}$, as well as above $\lambda>6 \mu \mathrm{m}$.

In order to validate the experimental measurement of diffuse reflection, the measured and computed reflectivity as a function of the scattering angle $\theta_{\text {out }}$ is plotted for an incident angle $\theta_{\text {in }}=50^{\circ}$ at a $\lambda=5.15 \mu \mathrm{m}$ wavelength for the periodic grating (red line and crosses) and the disordered array (blue line and crosses). As 
in Fig. 2(b), the numerical simulation is done for a focused beam $\left(d \theta=10^{\circ}\right)$, which leads to a pretty good agreement for both samples. There are three peaks for the periodic grating corresponding to the zeroth order $\left(\theta_{\text {out }}=50^{\circ}\right)$, the first order $\left(\theta_{\text {out }}=0^{\circ}\right)$ and the second order $\left(\theta_{\text {out }}=-40^{\circ}\right)$ of diffraction. Away from these angles, there is no scattered energy. In contrast, the disordered array scatters energy on each angle in the range $\theta_{\text {out }}$ between $-90^{\circ}$ and $40^{\circ}$, and with higher values near $\theta_{\text {out }}=0^{\circ}$, which is consistent with the single nanoantenna behavior. The absence of scattering for $\theta_{\text {out }}>60^{\circ}$ is confirmed by the computation, and is also not observed for scattering of a single nanoantenna. This intriguing behavior is present for other incident angles, and could be attributed to the disorder itself. It could open some interesting prospective such as producing diffusion only on a given range of angles.

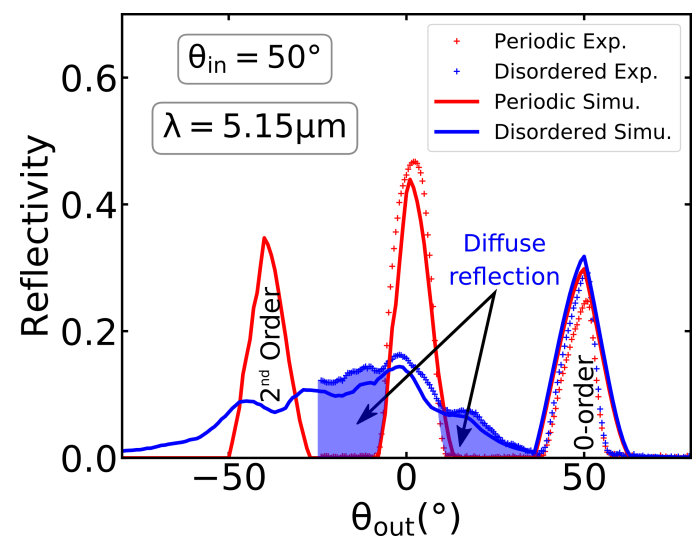

FIG. 5. Measured (continuous lines) and computed (crosses) reflectivity efficiency as a function of the scattering angle $\theta_{\text {out }}$ for an incidence angle $\theta_{\text {in }}=50^{\circ}$ at a $\lambda=5.15 \mu \mathrm{m}$ wavelength for the periodic grating (red line and crosses) and the disordered array (blue line and crosses).

Diffractive arrays of 1D-MIM nanoantennas have been studied both theoretically and experimentally. Theoretical predictions show that introducing disorder involves redistribution of the energy from diffraction mainly to diffuse reflection. Therefore, it is possible to change a highly diffractive periodic structure into a highly diffusive one only by changing the spatial distribution, these changes have nearly no influence on the absorption and zero-order reflectivity efficiencies. Our study highlights the importance of taking into account scattering of light as soon as random assemblies of random assemblies of plasmonic resonators are at stake, and this, even if localized resonances are involved. These results also pave the way to the design of metasurfaces for manipulating the scattering of light on a limited spectral range.

\section{ACKNOWLEDGEMENT}

This work has been supported by a DGA-MRIS scholarship.

${ }^{1}$ F. Ding, Y. Chen, and S. I. Bozhevolnyi, Applied Sciences 8, 594 (2018).

${ }^{2}$ P. Chevalier, P. Bouchon, J. Jaeck, D. Lauwick, N. Bardou, A. Kattnig, F. Pardo, and R. Haïdar, Applied Physics Letters 107, 251108 (2015).

${ }^{3}$ H. Bertin, Y. Brule, G. Magno, T. Lopez, P. Gogol, L. Pradere,

B. Gralak, D. Barat, G. Demesy, and B. Dagens, ACS photonics 5, 2661 (2018).

${ }^{4}$ A. Moreau, C. Ciracì, J. J. Mock, R. T. Hill, Q. Wang, B. J. Wiley, A. Chilkoti, and D. R. Smith, Nature 492, 86 (2012).

${ }^{5}$ G. M. Akselrod, J. Huang, T. B. Hoang, P. T. Bowen, L. Su, D. R. Smith, and M. H. Mikkelsen, Advanced Materials 27, 8028 (2015).

${ }^{6}$ J. Hao, R. Niemiec, L. Burgnies, E. Lheurette, and D. Lippens, Journal of Applied Physics 119, 193104 (2016).

${ }^{7}$ Y. Zhai, Y. Ma, S. N. David, D. Zhao, R. Lou, G. Tan, R. Yang, and X. Yin, Science 355, 1062 (2017).

${ }^{8}$ M. Albooyeh, S. Kruk, C. Menzel, C. Helgert, M. Kroll, A. Krysinski, M. Decker, D. N. Neshev, T. Pertsch, C. Etrich, et al., Scientific reports 4, 4484 (2014).

${ }^{9}$ K. Sasihithlu, N. Dahan, J.-P. Hugonin, and J.-J. Greffet, Journal of Quantitative Spectroscopy and Radiative Transfer 171, 4 (2016).

${ }^{10}$ T. Tani, S. Hakuta, N. Kiyoto, and M. Naya, Optics Express 22, 9262 (2014).

${ }^{11}$ M. Jang, Y. Horie, A. Shibukawa, J. Brake, Y. Liu, S. M. Kamali, A. Arbabi, H. Ruan, A. Faraon, and C. Yang, Nature photonics 12, 84 (2018)

${ }^{12}$ M. Dupré, L. Hsu, and B. Kanté, Scientific reports 8, 7162 (2018).

${ }^{13}$ A. Jouanin, J. P. Hugonin, and P. Lalanne, Advanced Functional Materials 26, 6215 (2016).

${ }^{14}$ T. J. Antosiewicz and T. Tarkowski, ACS Photonics 2, 1732 (2015).

${ }^{15}$ W.-B. Shi, L.-Z. Liu, R. Peng, D.-H. Xu, K. Zhang, H. Jing, R.H. Fan, X.-R. Huang, Q.-J. Wang, and M. Wang, Nano letters 18, 1896 (2018).

${ }^{16}$ Y. Nishijima, J. B. Khurgin, L. Rosa, H. Fujiwara, and S. Juodkazis, Optics Express 21, 13502 (2013).

${ }^{17}$ Z. Fusco, R. Bo, Y. Wang, N. Motta, H. Chen, and A. Tricoli, Journal of Materials Chemistry C (2019).

${ }^{18}$ L.-H. Gao, Q. Cheng, J. Yang, S.-J. Ma, J. Zhao, S. Liu, H.-B. Chen, Q. He, W.-X. Jiang, H.-F. Ma, et al., Light: Science \& Applications 4, e324 (2015).

${ }^{19}$ A. Pors, F. Ding, Y. Chen, I. P. Radko, and S. I. Bozhevolnyi, Scientific reports 6, 28448 (2016).

${ }^{20}$ J. Hao, J. Wang, X. Liu, W. J. Padilla, L. Zhou, and M. Qiu, Applied Physics Letters 96, 251104 (2010).

${ }^{21}$ C. Koechlin, P. Bouchon, F. Pardo, J. Jaeck, X. Lafosse, J. Pelouard, and R. Haidar, Applied Physics Letters 99, 241104 (2011).

${ }^{22}$ P. Bouchon, C. Koechlin, F. Pardo, R. Haïdar, and J. Pelouard, Optics Letters 37, 1038 (2012).

${ }^{23}$ C. Koechlin, P. Bouchon, F. Pardo, J.-L. Pelouard, and R. Haïdar, Optics Express 21, 7025 (2013).

${ }^{24}$ Y. Cui, Y. He, Y. Jin, F. Ding, L. Yang, Y. Ye, S. Zhong, Y. Lin, and S. He, Laser \& Photonics Reviews 8, 495 (2014).

${ }^{25}$ R. Esteban, T. V. Teperik, and J.-J. Greffet, Physical Review Letters 104, 026802 (2010).

${ }^{26}$ M. Makhsiyan, P. Bouchon, J. Jaeck, J.-L. Pelouard, and R. Haïdar, Applied Physics Letters 107, 251103 (2015).

${ }^{27}$ C. Li, V. Krachmalnicoff, P. Bouchon, J. Jaeck, N. Bardou, R. Haïdar, and Y. De Wilde, Physical Review Letters 121, 243901 (2018). 
${ }^{28}$ F. Marquier, J.-J. Greffet, S. Collin, F. Pardo, and J. Pelouard, Optics Express 13, 70 (2005).

${ }^{29}$ P. Jouy, Y. Todorov, A. Vasanelli, R. Colombelli, I. Sagnes, and C. Sirtori, Applied Physics Letters 98, 021105 (2011).

${ }^{30}$ P. Bouchon, F. Pardo, R. Haïdar, and J. Pelouard, Journal of the Optical Society of America A 27, 696 (2010).
${ }^{31}$ P. Chevalier, P. Bouchon, F. Pardo, and R. Haïdar, Journal of the Optical Society of America A 31, 1692 (2014).

${ }^{32}$ E. D. Palik and G. Ghosh, Handbook of optical constants of solids (Academic press, 1985).

${ }^{33}$ J. Kischkat, S. Peters, B. Gruska, M. Semtsiv, M. Chashnikova, M. Klinkmüller, O. Fedosenko, S. Machulik, A. Aleksandrova, G. Monastyrskyi, et al., Applied optics 51, 6789 (2012). 